\title{
Ataxia, tremor, intellectual disability: a case of STXBP1 encephalopathy with a new mutation
}

\author{
Aydan Değerliyurtt ${ }^{1}$, Gamze Gezgen Kesen², Serdar Ceylaner ${ }^{3}$ \\ Departments of ${ }^{1}$ Pediatric Neurology and ${ }^{2}$ Pediatrics, Ankara Children's Hematology and Oncology Training and Research \\ Hospital; ${ }^{3}$ Intergen Genetics Centre, Ankara, Turkey. E-mail:degerliyurta@gmail.com \\ Received: 14th July 2018, Revised: 24th September 2018, 18th November 2018, \\ Accepted: 20th November 2018
}

SUMMARY: Değerliyurt A, Gezgen Kesen G, Ceylaner S. Ataxia, tremor, intellectual disability: a case of STXBP1 encephalopathy with a new mutation. Turk J Pediatr 2019; 61: 757-759.

\begin{abstract}
STXBP1 gene mutations are among the most common mutations in earlyonset epileptic encephalopathies. The clinical spectrum of STXBP1 mutations is not limited to epileptic phenotypes and also includes atypical Rett syndrome and non-syndromic sporadic severe intellectual disability. Tremor, dystonia, choreiform movements, stereotypical head movements and ataxia may also be seen. However, the phenotypical spectrum is not as well-known as the other common SCN1A or CDKL5 gene mutations, making the clinical diagnosis difficult and usually requiring gene panel studies or whole exome sequencing for the diagnosis. We present a 17-year-old male patient whose seizures started at the age of 12 years. The patient could only make limited eye contact, would continuously scream, and also had severe intellectual disability, marked ataxic walking and a very significant coarse tremor. The patient was clinically thought to have STXBP1 encephalopathy due to the presence of severe intellectual disability together with tremor, and ataxia. STXBP1 gene analysis revealed a new c.9_13delCATTG (pIle4Profs*12) (p.I4Pfs*12) (heterozygous) frameshift mutation. In conclusion, STXBP1 encephalopathy should be considered if severe intellectual disability is accompanied by severe tremor and ataxia in a patient with epileptic and developmental encephalopathy. A normal head circumference supports the diagnosis in such patients.
\end{abstract}

Key words: STXBP1, epileptic encephalopathy, ataxia, tremor, intellectual disability.

STXBP1 (MUNC18-1) is an indispensable protein that plays a significant role in synaptic vesicle development and fusion and the related neurotransmitter secretion which is widely found in the brain. ${ }^{1,2}$ STXBP1 haploinsufficiency causes prolongation of the synaptic depression at all synapses and especially GABAergic ones, resulting in hyperexcitability and epileptic activity. ${ }^{1}$ STXBP1 gene mutations have been primarily shown in Ohtahara syndrome patients with early-onset encephalopathy. ${ }^{3}$ The mutation spectrum was later extended to the West syndrome, unclassified early-onset epileptic encephalopathy, Dravet syndrome, intellectual disability not accompanied by epilepsy, autism, and Rett syndrome. ${ }^{4}$ Although not reported in the initial cases due to the authors focus on epilepsy in general, movement disorders such as stereotypic, dyskinetic and choreiform movements, tremor episodes, dystonic posture, rhythmic extremity movements, short axial contractions and stereotypic head movements were also later shown to be associated with STXBP1 encephalopathy in various age groups. ${ }^{5-7}$ Despite being one of

The case was presented as a poster at the 20th Annual Congress of the Turkish-Child Neurology Association -that took place on 2-6 May 2018-, at the Turkish Republic of Northern Cyprus, and was published as an abstract in the abstract book. 
the most common mutations, patients with STXBP1 encephalopathy are not as easily diagnosed as patients with the SCN1A or CDKL5 mutation as its phenotypical spectrum is not known as well.

We present a patient who was referred with epilepsy and was then found to have a new STXBP1 mutation with genetic analysis conducted after the triad of severe intellectual disability, tremor, and ataxia was detected. We emphasize that this triad is an important clue in the diagnosis of STXBP1 encephalopathy, especially in older children who can walk.

\section{Case Report}

A 17-year-old male who had previously been followed-up at an external center for autism was brought from the nursing home for generalized tonic-clonic seizures continuing for 1-2 minutes for the last 10 days. The records revealed that the patient had first presented to our hospital at the age of 12 years with newly occurring seizures and rapid breathing when excited and agitation, the brain MRI had been normal other than mega cisterna magna at the time. Electroencephalography (EEG) had been requested but could not be performed and he had been lost to follow-up for five years. We found out that the patient was born at a weight of 3600 gr from parents who were not consanguineous and that he had not needed hospital care during the neonatal period. He had started walking at the age of two years and began talking at the age of four years. He could only speak a few words. The examination at the last presentation revealed a head circumference of $58 \mathrm{~cm}+2 \mathrm{SD}$, an autistic appearance, limited eye contact, screaming, repeating meaningless sounds, and hyperactivity. He did not react to touch during the examination and showed no aggressive behavior. His walk was ataxic and there was a widespread coarse tremor of the upper extremities that was prominent and increased with movement. The blood count, electrolytes, thyroid function tests, creatine kinase, B12 vitamin level, and the detailed metabolic investigation (urine and blood amino acids, carnitine-acyl carnitine measurement with tandem mass, urine organic acid analysis), and echocardiography results were normal. The
MRI was repeated and no difference with the previous scan was found. Epileptiform activity was observed in the bilateral frontal regions in the EEG and levetiracetam was added to the current valproate and lamotrigine treatment. The presence of tremor and ataxia together with the severe intellectual disability resulted in the consideration of STXBP1 encephalopathy. Following written consent from the institution that served as the legal guardian of the patient, an STXBP1 gene mutation study was requested. Sequence analysis of the STXBP1 gene was conducted using a next generation sequencing system (MiseqIllumina- San Diego) with in house designed primers, resulting in the detection of the heterozygote NM_003165.3:c.9_13delCATTG (p.Ile4Profs*12) (p.I4Pfs*12) mutation. This variant is classified as a pathogenic variant according to ACMG criteria as it is a null variant (frameshift mutation) and the allele was not found in the Broad gnomAD exomes and Broad gnomAD genomes. Informed consent was obtained from the family and the institution that served as the legal guardian of the patient.

\section{Discussion}

We found a new mutation in the STXBP1 gene in a patient with late-onset epilepsy, ataxia, severe tremor and autistic symptoms such as limited eye contact, rapid breathing, and screaming. While our patient had originally been followed-up for developmental delay and autism, his initial attacks had started when he was 12 years old. In the largest series of patients with the STXBP1 mutation, epilepsy was found in $94 \%$, and late-onset epilepsy as in our patient in 5\% (8/147 patients), and intellectual disability without epilepsy in $6 \%$ (9/147 patients). ${ }^{4}$ This suggests that STXBP1 mutations should not only be considered in patients with early-onset epileptic encephalopathy but also in the differential diagnosis in patients with developmental encephalopathy and late-onset epilepsy and even in those with no epilepsy.

Although autistic symptoms were present in our patient, there was no aggressive behavior or anxiety response to touching. Autistic signs have been reported in $30 \%$ of the patients 
specifically queried regarding such symptoms and in $20 \%$ of the patients not specifically queried in the Stamberger et al. ${ }^{4}$ study.

The most important findings of our patient that led to the diagnosis were the marked tremor and ataxia. A significant tremor with high amplitude that appeared especially during voluntary movements was present. Ataxia and tremor were reported in about 20\% of the largest series of patients. ${ }^{4}$ Di Meglio et al. ${ }^{8}$ reported that $29 \%$ of their patients could walk, usually in an ataxic manner. Ataxia was also reported in the patients who could walk by Deprez et al. ${ }^{9}$ Gburek-Augustat et al. ${ }^{10}$ reported three patients who were diagnosed in childhood (7, 11 and 11.5 years) with ataxia, tremor and intellectual disability but no epilepsy.

As far as we know, our case is the second patient (after case 3 of the Gburek-Augustat et al. ${ }^{10}$ study) to be clinically diagnosed with the STXBP1 mutation with ataxia, tremor and retardation in the literature. We therefore believe the presence of ataxia and tremor in children with severe intellectual disability who can walk is an important indicator that STXBP1 gene analysis may be required, whether epilepsy is present or not.

The head circumference in our patient was above +2SD despite the severe intellectual disability. Even if early-onset epilepsy is present, a normal head circumference seems to be an important characteristic in patients with the STXBP1 gene mutation. ${ }^{8}$

In conclusion, STXBP1 encephalopathy has been defined quite recently and its phenotypic spectrum is still being identified with newly reported cases and mutations. A new clinical phenotype of STXBP1 encephalopathy should be considered if severe tremor and ataxia is present in a patient with severe-intellectual disability, whether accompanied by epilepsy or not. A normal head circumference supports the diagnosis in such patients.

\section{REFERENCES}

1. Toonen RF, Wierda K, Sons MS, et al. Munc181 expression levels control synapse recovery by regulating readily releasable pool size. Proc Natl Acad Sci U S A 2006; 103: 18332-18337.

2. Gerber SH, Rah JC, Min SW, et al. Conformational switch of syntaxin-1 controls synaptic vesicle fusion. Science 2008; 321: 1507-1510.

3. Saitsu H, Kato M, Mizuguchi $T$, et al. De novo mutations in the gene encoding STXBP1 (MUNC181) cause early infantile epileptic encephalopathy. Nat Genet 2008; 40: 782-788.

4. Stamberger $\mathrm{H}$, Nikanorova $\mathrm{M}$, Willemsen $\mathrm{MH}$, et al. STXBP1 encephalopathy: A neurodevelopmental disorder including epilepsy. Neurology 2016; 86: 954962.

5. Milh M, Villeneuve N, Chouchane M, et al. Epileptic and nonepileptic features in patients with early onset epileptic encephalopathy and STXBP1 mutations. Epilepsia 2011; 52: 1828-1834.

6. Mignot C, Moutard ML, Trouillard O, et al. STXBPrelated encephalopathy presenting as infantile spasms and generalized tremor in three patients. Epilepsia 2011; 52: 1820-1827.

7. Kim YO, Korff CM, Villaluz MMG, et al. Head stereotypies in STXBP1 encephalopathy. Dev Med Child Neurol 2013; 55: 769-772.

8. Di Meglio C, Lesca G, Villeneuve N, et al. Epileptic patients with de novo STXBP1 mutations: Key clinical features based on 24 cases. Epilepsia 2015; 56: 1931-1940.

9. Deprez L, Weckhuysen S, Holmgren P, et al. Clinical spectrum of early-onset epileptic encephalopathies associated with STXBP1 mutations. Neurology 2010; 75: 1159-1165.

10. Gburek-Augustat J, Beck-Woedl S, Tzschach A, Bauer $\mathrm{P}$, Schoening M, Riess A. Epilepsy is not a mandatory feature of STXBP1 associated ataxia-tremorretardation syndrome. Eur J Paediatr Neurol 2016; 20: 661-665. 